\title{
Application of ground scatter returns for calibration of HF interferometry data
}

\author{
Pavlo Ponomarenko ${ }^{1,3^{*}}$, Nozomu Nishitani ${ }^{1}$, Alexey V. Oinats ${ }^{2}$, Taishi Tsuya ${ }^{1}$ and Jean-Pierre St.-Maurice ${ }^{3}$
}

\begin{abstract}
Information on the vertical angle of arrival (elevation) is crucial in determining propagation modes of high-frequency $(\mathrm{HF}, 3-30 \mathrm{MHz}$ ) radio waves travelling through the ionosphere. The most advanced network of ionospheric HF radars, SuperDARN (Super Dual Auroral Radar Network), relies on interferometry to measure elevation, but this information is rarely used due to intrinsic difficulties with phase calibration as well as with the physical interpretation of the measured elevation patterns. In this work, we propose an empirical method of calibration for SuperDARN interferometry. The method utilises a well-defined dependence of elevation on range of ground scatter returns. "Fine tuning" of the phase is achieved based on a detailed analysis of phase fluctuation effects at very low elevation angles. The proposed technique has been successfully applied to data from the mid-latitude Hokkaido East SuperDARN radar. It can also be used at any other installation that utilises HF interferometry.
\end{abstract}

Keywords: Ionospheric radio wave propagation; High-frequency radars; Interferometry

\section{Background}

High-frequency (HF, $10-20 \mathrm{MHz}$ ) radars are actively used for monitoring ionospheric conditions at high and mid-latitudes and provide information on plasma dynamics (drifts, diffusion etc.) at E- and F-region heights. Currently, the most advanced instrument in this category is the Super Dual Auroral Radar Network (SuperDARN) which covers high to mid-latitude regions in both hemispheres (Greenwald et al. 1995, Chisham et al. 2007). SuperDARN typically consists of pairs of radars with overlapping fields of view which allows for the estimate of a horizontal vector of ionospheric plasma drift based on the Doppler frequency shift of the ionospheric backscatter returns. This information is used for the reconstruction of ionospheric electric potential at F-region heights (Ruohoniemi and Baker 1998).

One of the most important tasks in the interpretation of the HF echoes is to establish their propagation mode so that the Doppler shift information can be correctly converted into electric field. Among the multitude of the HF propagation modes existing in the ionosphere, the conventional software distinguishes only between the

\footnotetext{
* Correspondence: pasha.ponomarenko@usask.ca

${ }^{1}$ Solar-Terrestrial Environment Laboratory, Nagoya University, Nagoya, Japan

${ }^{3}$ University of Saskatchewan, Saskatoon, SK, Canada

Full list of author information is available at the end of the article
}

ionospheric and ground scatter (Blanchard et al. 2009). Much more detailed and accurate propagation information can be obtained from the dependence of elevation angle as a function of range (e.g. Kelso 1964). These data are actually available for most of the SuperDARN radars, for which elevation is estimated from the phase delay between two spatially separated antenna arrays (Greenwald et al. 1995, Milan et al. 1997). And yet, while the elevation data have been recorded since the inception of SuperDARN, they have rarely been used until recently due to inherent technical difficulties related to the phase calibration of the HF arrays, e.g. using a standard source located at a fixed elevation. Furthermore, while the phase offset between the interferometer arrays has to be carefully reevaluated after each hardware and software update, it is also a subject to gradual changes in the physical properties of the antennas, cables, and electric circuitry. Therefore, there is a pressing need in a simple but reliable way of monitoring these changes.

In this work, we lay the physical and statistical foundations for empirically estimating the phase offsets in SuperDARN data. The proposed approach was thoroughly tested and then applied to Hokkaido East data allowing for the physically justified estimation of the elevation, which considerably extends diagnostic capabilities of the network. 


\section{Methods}

\section{SuperDARN interferometry}

The main array of SuperDARN radars is used both for radio wave emission and reception of echoes. It contains a line of 16 elements (either log-periodic director antennas or broad-band wire dipoles), which are separated by $\approx 15 \mathrm{~m}$. The horizontal alignment of the array elements produces a knife-like diagram which is relatively narrow in the azimuthal plane and broad in the vertical plane. Scanning (beam-forming) in azimuth is achieved by applying a linear phase shift between array elements. Usually, SuperDARN arrays scan consecutively through 16 azimuthal directions (beams) within $\pm 27^{\circ}$ from the boresight direction. Elevation selection is based on measuring phase shift $\Psi$ between the echoes received by the main array and an auxiliary (interferometer) array. The latter consists of four elements and is usually located at $100 \mathrm{~m}$ in front of or behind the main array (Fig. 1). Both arrays form beams pointing in the same azimuthal direction so that the narrower beam from the main array is "embedded" into the wider interferometer beam. The elevation angle is calculated as $\Delta=$ $\cos ^{-1}[\Psi / k d \cos \phi]$, where $k=2 \pi / \lambda$ is the radar wave vector magnitude, $d$ is the interferometer base and $\phi$ is azimuth measured from the boresight direction (Milan et al. 1997).

There is an intrinsic ambiguity arising from the fact that the interferometer base of $d=100 \mathrm{~m}$ is larger than the radar wavelength, $\lambda \approx 20-30 \mathrm{~m}$. As a result, the maximum phase shift (observed at zero elevation), $\Psi_{\max }=k d$ $\cos \phi$, lies between $6 \pi$ and $10 \pi$, while instrumentally the phase can only be measured between $-\pi$ and $+\pi$. Figure 2 shows the relationship between elevation and phase shift for central beams $(\phi=0)$ at the typical Hokkaido East frequency of $11 \mathrm{MHz}$. The black line shows the total phase shift while the blue lines correspond to the actually measured phase shift which is confined to within $\pm \pi$ range. The latter illustrates the above-mentioned ambiguity with the same phase shift corresponding to multiple elevation values.
The ionospheric scatter echoes are expected to come from anisotropic plasma irregularities aligned with the geomagnetic field lines, and the maximum backscatter power is observed when the radio wave propagates in the direction orthogonal to the field lines. At high latitudes, the field lines are nearly vertical so that the backscatter elevation is expected to be closer to the horizontal direction, somewhere between 0 and $30^{\circ}-40^{\circ}$. As a result, in SuperDARN software, the assumption made that the echoes come from within the first segment, i.e. between $\Psi_{\max }-2 \pi$ and $\Psi_{\max }$, and the resulting elevation estimates lie between zero and some maximum value $\Delta_{2 \pi}=\cos ^{-1}\left[\left(\Psi_{\max }-2 \pi\right) / \Psi_{\max }\right]$ (Milan et al. 1997). This phase shift range is highlighted by red in Fig. 2.

\section{Theoretical dependence of elevation vs range for ground scatter}

In order to estimate a phase offset, first we need to identify an expected pattern for a known propagation mode of the radio wave. In order to produce plasma circulation maps, SuperDARN utilises HF backscatter returns from small-scale ionospheric irregularities (ionospheric scatter, IS). Importantly, spatio-temporal characteristics of IS are subject to different kinds of plasma instabilities which may or may not be present in the radar's field-ofview, in contrast to the regular ground scatter (GS) echoes, which represent radio waves "reflected" by the regular ionospheric layer before they are scattered back by the rugged ground surface. While GS echoes are treated as interference by SuperDARN data processing procedures, their regular character makes this propagation mode very useful as a reference in analysing interferometer phase patterns.

Figure 3 represents a ray tracing simulation of $\mathrm{HF}$ propagation in a simplified situation of a single Chapman layer with a maximum density located at $300-\mathrm{km}$ altitude and a scale height of $30 \mathrm{~km}$. The ray tracing code was developed by Ponomarenko et al. (2009) and utilises Snell's law and the simplest form of the Appleton-Hartree

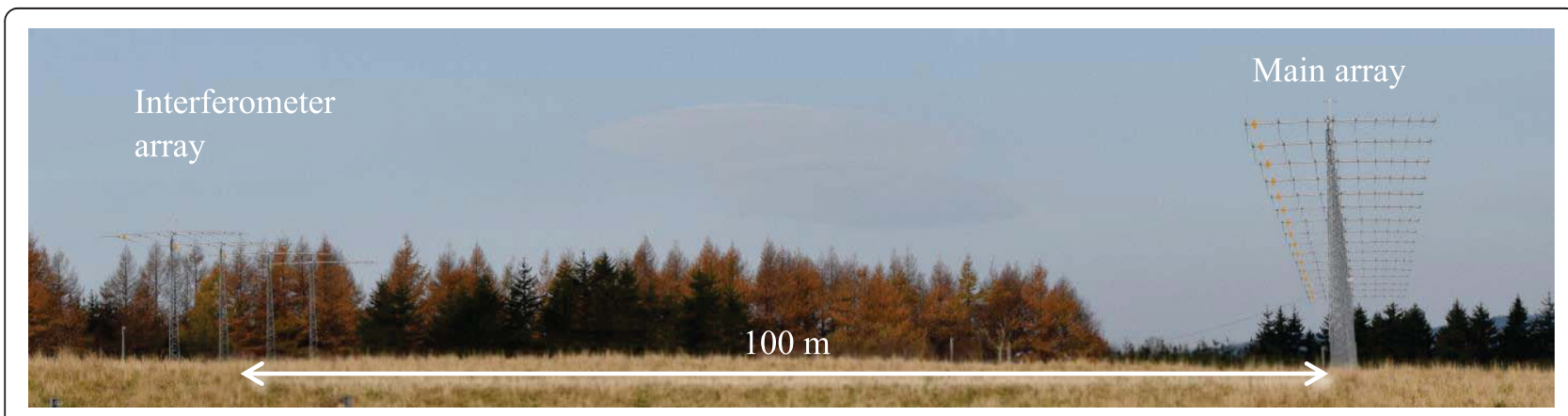

Fig. 1 Hokkaido East SuperDARN radar antennas 


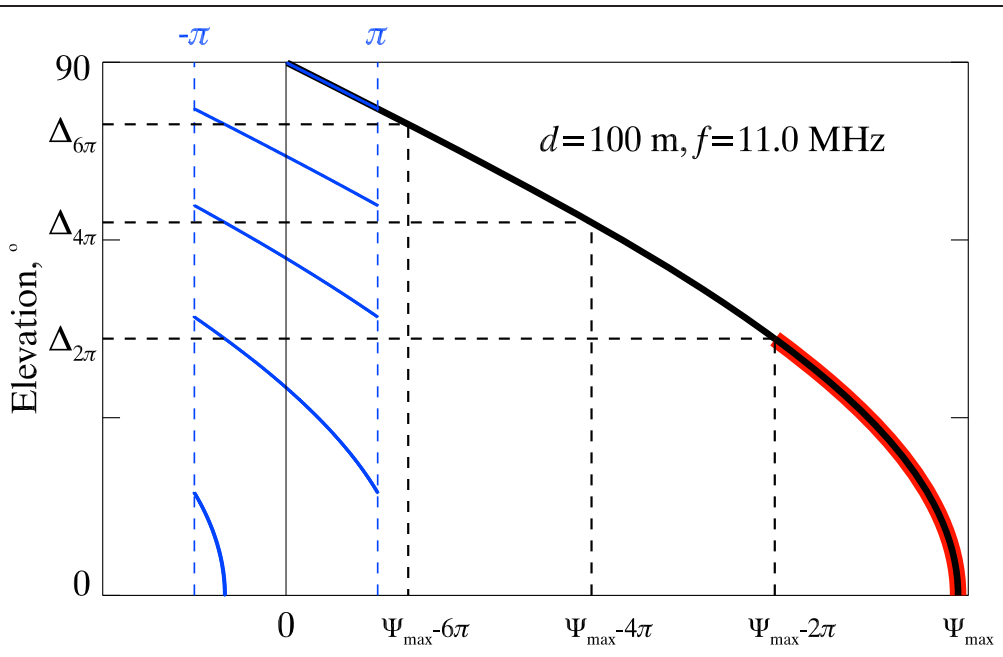

Phase shift, rad

Fig. 2 Transfer function between interferometer phase shift and elevation angle obtained for the boresight direction of the radar antenna. The black curve shows total phase shift for the given interferometer base of $100 \mathrm{~m}$ and a typical Hokkaido radar frequency of $11 \mathrm{MHz}$ for the elevation range between $0^{\circ}$ and $90^{\circ}$ (for the remaining back-lobe range $90^{\circ}-180^{\circ}$, the dependence should be mirrored around $90^{\circ}$ elevation and zero phase). The blue line corresponds to the measured phase confined to the $\pm \pi$ interval and shows that a single measured phase value corresponds to multiple elevation angles. The conventional $2 \pi$ range used for calculating SuperDARN elevation is highlighted by red

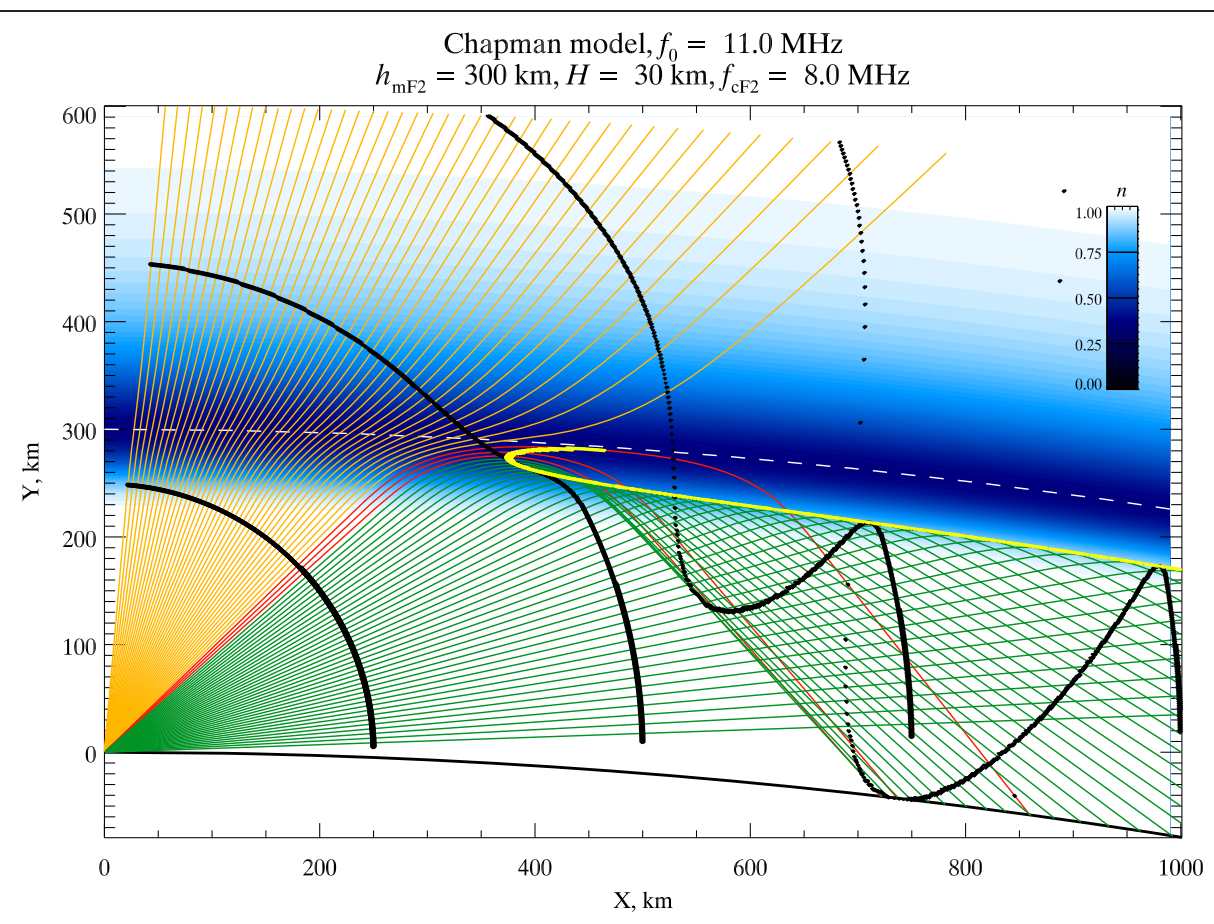

Fig. 3 Numerical ray-tracing simulation of an 11-MHz radio wave propagating through a horizontally uniform Chapman layer with the maximum height of $300 \mathrm{~km}$, the scale height of $30 \mathrm{~km}$ and the maximum plasma frequency of $8 \mathrm{MHz}$. The orange, red and green trajectories show escaping, Pedersen (high-angle) and low-angle rays, respectively. The black dots show group range marks at 250-km steps. The yellow contour shows "reflection" point locations for the Pedersen and the low-angle rays. The blue shading illustrates distribution of the ionospheric refractive index 
equation, $n^{2}=1-f_{p}^{2} / f_{0}^{2}$, where $f_{0}$ and $f_{\mathrm{p}}$ are the wave frequency and plasma frequency, respectively. The ray trajectories were simulated between $0^{\circ}$ and $85^{\circ}$ separated by $0.1^{\circ}$ in elevation at the radar location. The physical range resolution is $1 \mathrm{~km}$ (in Fig. 3, the rays are plotted at $1^{\circ}$ intervals only). The blue shading shows the spatial distribution of the ionospheric refractive index. The black dots show group range in $250-\mathrm{km}$ steps. There are three major propagation modes: (i) low-angle rays (green), (ii) high-angle (Pedersen) rays (red), and (iii) escaping rays (orange). While all three propagation modes can contribute to IS, only the first two reach the ground and are capable of generating GS. The yellow contour corresponds to the turning ("reflection") points of the rays with the top and bottom branches produced by the Pedersen and lowangle rays, respectively, while their convergence point at close ranges corresponds to the skip zone boundary on the ground. The Pedersen mode covers a narrow angular range $\left(\approx 3^{\circ}\right.$ in this case) and corresponds to divergent rays "gliding" along the ionospheric maximum. This contrasts with the low-angle mechanism covering a comparatively large angular range and producing significantly larger power density due to convergent rays "reflected" from the bottom part of the ionospheric layer.

As a consequence of the larger ray/power density, the low-ray mode is expected to dominate GS returns. This assumption is supported by analysing simulated GS elevation at each $45-\mathrm{km}$ range gate. The median value for all trajectories reaching the ground was estimated within each $45-\mathrm{km}$ range of group delays. The result is presented in the middle panel of Fig. 4. Here, different colours correspond to different maximum plasma frequencies increasing from 5 to $10 \mathrm{MHz}$ (dark blue to red) in $1-\mathrm{MHz}$ steps. The elevation values gradually decrease with increasing range until they approach zero level, as would be expected from the low-angle mode. The horizontal dash line shows the maximum measured elevation, $\Delta_{2 \pi}$. The top panel in Fig. 4 shows respective phase shift values. For convenience, the phase was shifted by the maximum possible phase value, namely $\left(\Psi-\Psi_{\max }\right)$, so that a smaller phase shift corresponds to a lower elevation and vice versa. The bottom panel shows the respective values of the virtual height calculated from the group range $r$ and elevation $\Delta$ accounting for the spherical geometry, $h_{v}=\sqrt{R_{E}^{2}+r^{2}+2 R_{E} r \sin \Delta}-R_{E}$, where $R_{E}$ is the Earth's radius (Andre et al. 1998). The virtual height decreases with distance at close ranges and then stays at an almost constant level. These "saturation" altitudes are lower for higher critical frequencies. The near-range cut-off represents an ionospheric projection of the skip zone boundary. This boundary moves away from the radar with decreasing $f_{\mathrm{mF} 2}$ because the radio wave gets "reflected" at a progressively lower elevation, subject to the secant law. The closest data point at the highest critical frequency $(10 \mathrm{MHz}$, red) has an elevation value that exceeds the maximum measured value of $\Delta_{2 \pi} \approx$ $43^{\circ}$, so its phase was adjusted by the radar software producing an artificially low value of $\Delta \approx 30^{\circ}$.

In order to estimate sensitivity of the obtained elevation patterns to the presence of the horizontal ionospheric gradients, we performed an additional simulation with an overall ionospheric density increase or decrease with range. For a realistic density gradient value of $30 \%$ per $1000 \mathrm{~km}$, there were only minor changes in the elevation patterns (not shown) which did not alter the overall decrease of elevation with range.

At the next stage, we investigated distortions to the elevation and virtual height patterns caused by an arbitrary phase offset, $\Delta \Psi$. In Fig. 5, the unperturbed values $(\Delta \Psi=0)$ for $f_{\mathrm{mF} 2}=9 \mathrm{MHz}$ are plotted by a solid black line, while the coloured diamonds correspond to different offset values ranging from 0 to $360^{\circ}$ in $60^{\circ}$ steps. The offset causes some phase values to go outside of the "allowed" range so these values are automatically brought inside the range by either adding or subtracting $2 \pi$. As a result, most of the phase curves in Fig. 5 exhibit a $2 \pi$ discontinuity which shifts to longer ranges with increasing $\Delta \Psi$. The general effect on elevation is that the retrieved angle, instead of following a monotonous dependence on range, is split into two seemingly unrelated populations. To the left from the phase discontinuity (closer ranges), elevation decreases faster compared to the situation with no offset, while to the right (farther ranges) the situation is the opposite. The most noticeable effect on the virtual height is that at farther ranges, instead of becoming nearly constant, the derived virtual height increases monotonically.

\section{Proposed calibration method}

Based on the simulation results, the first step would be to analyse if the experimental elevation-range dependences for GS match the expected dependence on range. If they show patterns similar to those in Fig. 5, i.e. with sharp phase jumps and increasing virtual height at larger ranges, then one would need to introduce an extra phase/time offset so that the patterns would look as those in Fig. 4. While this task seems to be simple, there are several critical points to consider.

The most important factor is a correct interpretation of the data uncertainties. Previous studies by Ponomarenko et al. (2011a) have demonstrated that the accuracy of the phase estimates is mainly limited by variations arising from the statistical nature of the radar returns. The same phase fluctuation levels lead to larger elevation errors at lower angles due to the strongly non-linear phase-elevation transfer function near $\Psi_{\max }$ (Fig. 2). At low elevation levels, the bulk of measured phase shift values will lie just below the maximum possible value, $\Psi_{\max }$, but the statistical spread in 

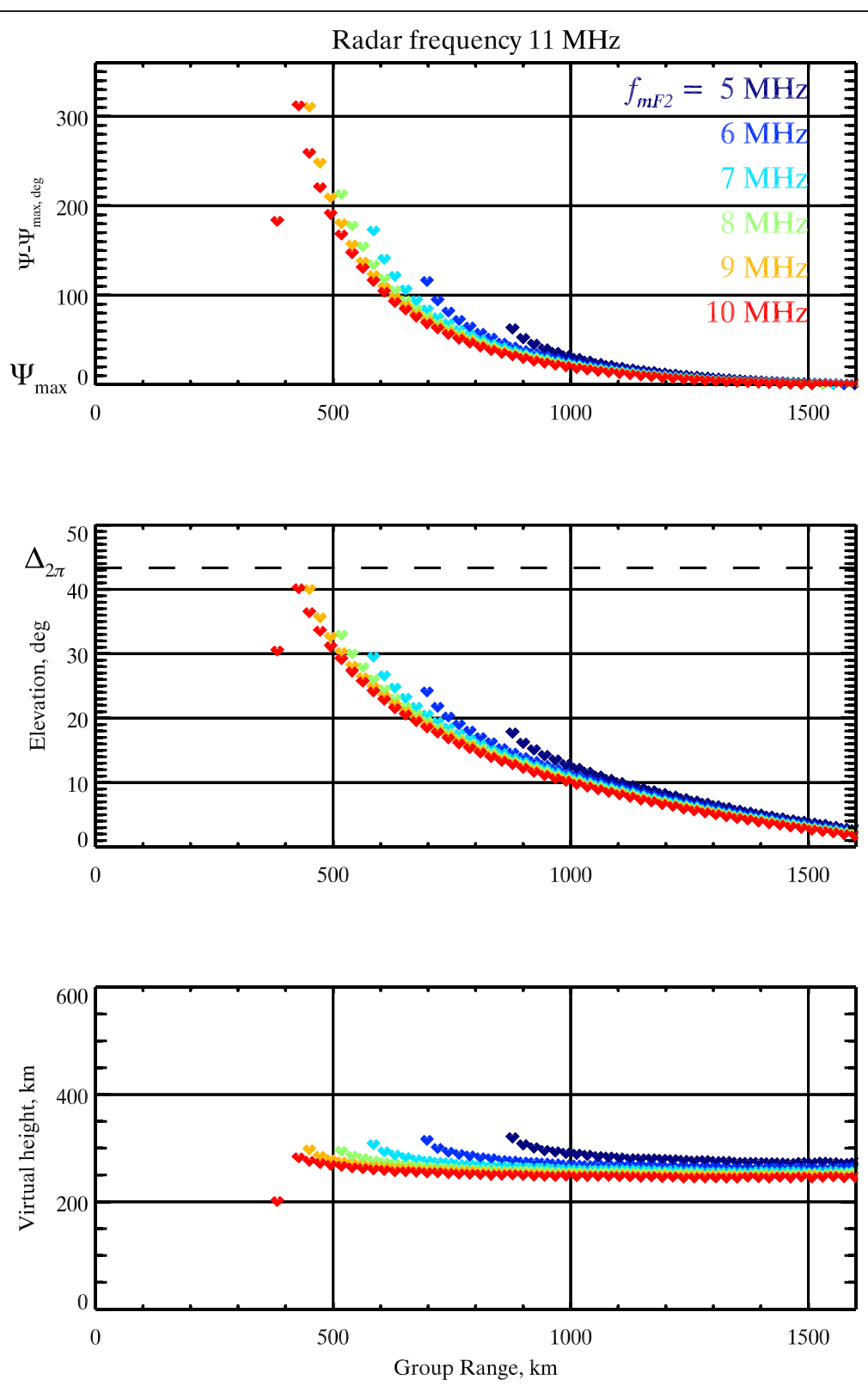

Fig. 4 Simulated phase shift (top), elevation (middle) and virtual height (bottom) variations vs group range for the reflection points of ground scatter in Fig. 3. The data were processed in the way that resembles operation of SuperDARN radars with a 45-km group range resolution (see text for more details)

the measured phase will shift some of the "true" phase values beyond $\Psi_{\max }$. The data processing software automatically subtracts $2 \pi$ from these values to shift them inside the "allowed" phase range so that the adjusted phase values become close to $\Psi_{\max }-2 \pi$, i.e. the respective elevation values are shifted to the maximum value corresponding to the first $2 \pi$ "wrap", $\Delta_{2 \pi}$. As a result, at far ranges (low elevation), even properly calibrated data should contain some sporadic discontinuities caused by the statistical variability of the phase measurements. However, on the range-time map, these discontinuities should present just isolated pixels of high elevation values on the predominantly low elevation background.

The above "statistical" discontinuities should not be confused with those observed when echo elevation truly exceeds $\Delta_{2 \pi}$. In contrast with the statistical error in the phase, the latter are automatically shifted to the lower elevation values. This sort of discontinuity is easy to recognise because it is generally located at closer ranges and represents a regular feature which is observed across 

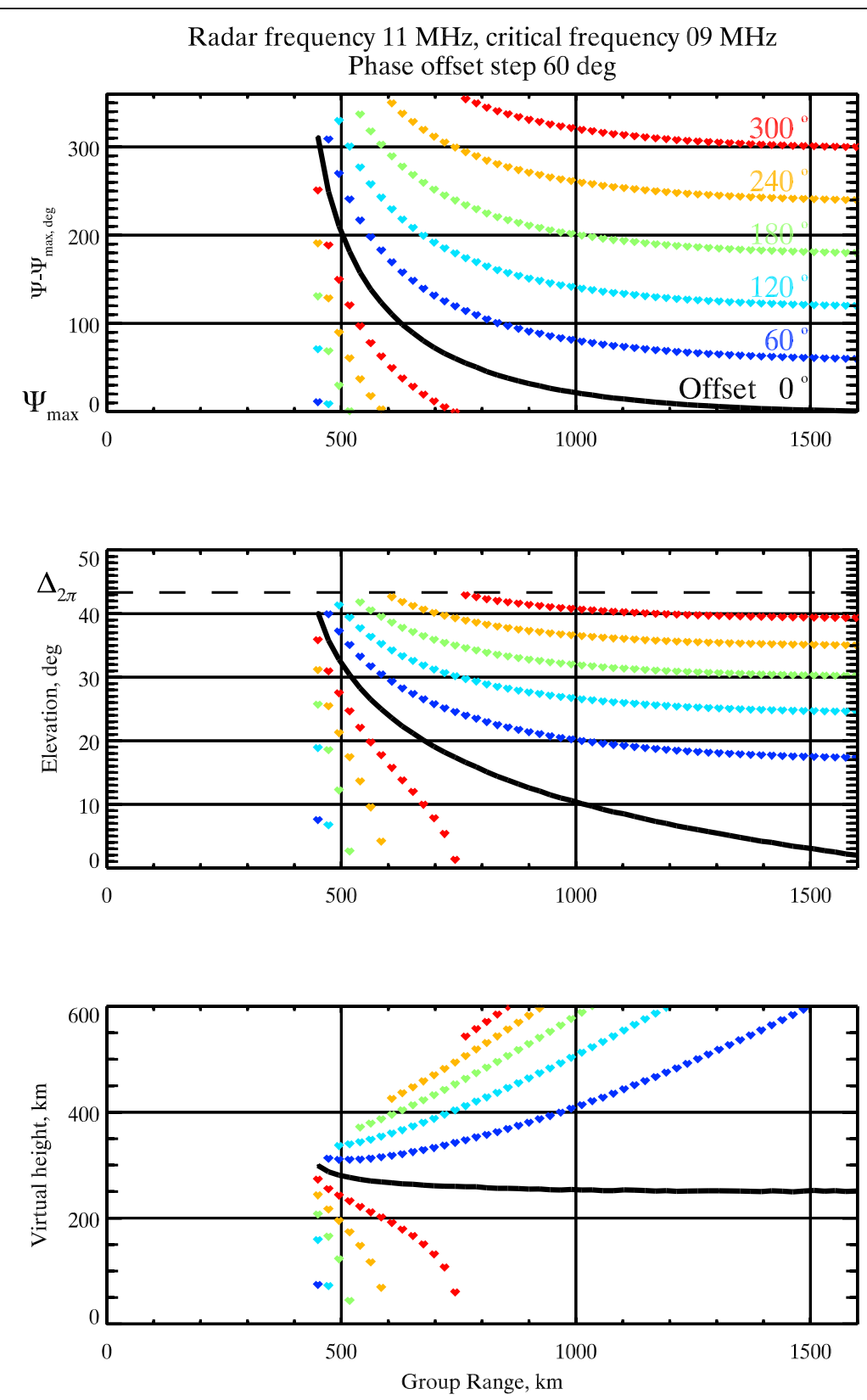

Fig. 5 Same as in Fig. 4 but for the fixed radar frequency of 9-MHz data with varying phase offset

consecutive scans. Most importantly, in this case, there will be no increase in the virtual height at progressively larger ranges.

Based on the above observations, we propose a new method to detect and measure the phase offset using visual analysis of the range-time elevation maps for ground scatter. The technique is based on consecutive adjustments of the interferometer phase until the elevation data show the expected pattern, i.e. a general decrease of elevation with range which shows at large distances a near-zero background accompanied by sporadic isolated "jumps" to values close to $\Delta_{2 \pi}$. Conveniently, in the hardware radar profile, there is a parameter $\operatorname{tdiff}$ which allows for phase adjustment in terms of time delay expressed in nanoseconds. At the time of publication, the Virginia Tech SuperDARN group (2015) provided an opportunity to perform the adjustment procedure by tuning tdiff and visually analysing the resulting elevation patterns.

\section{Results and discussion}

We illustrate the validity and effectiveness of our new calibration method by analysing a 6-h interval (06:0012:00UT, 23 February 2014) of the data from Hokkaido East SuperDARN radar as shown in Fig. 6. Here, the top 


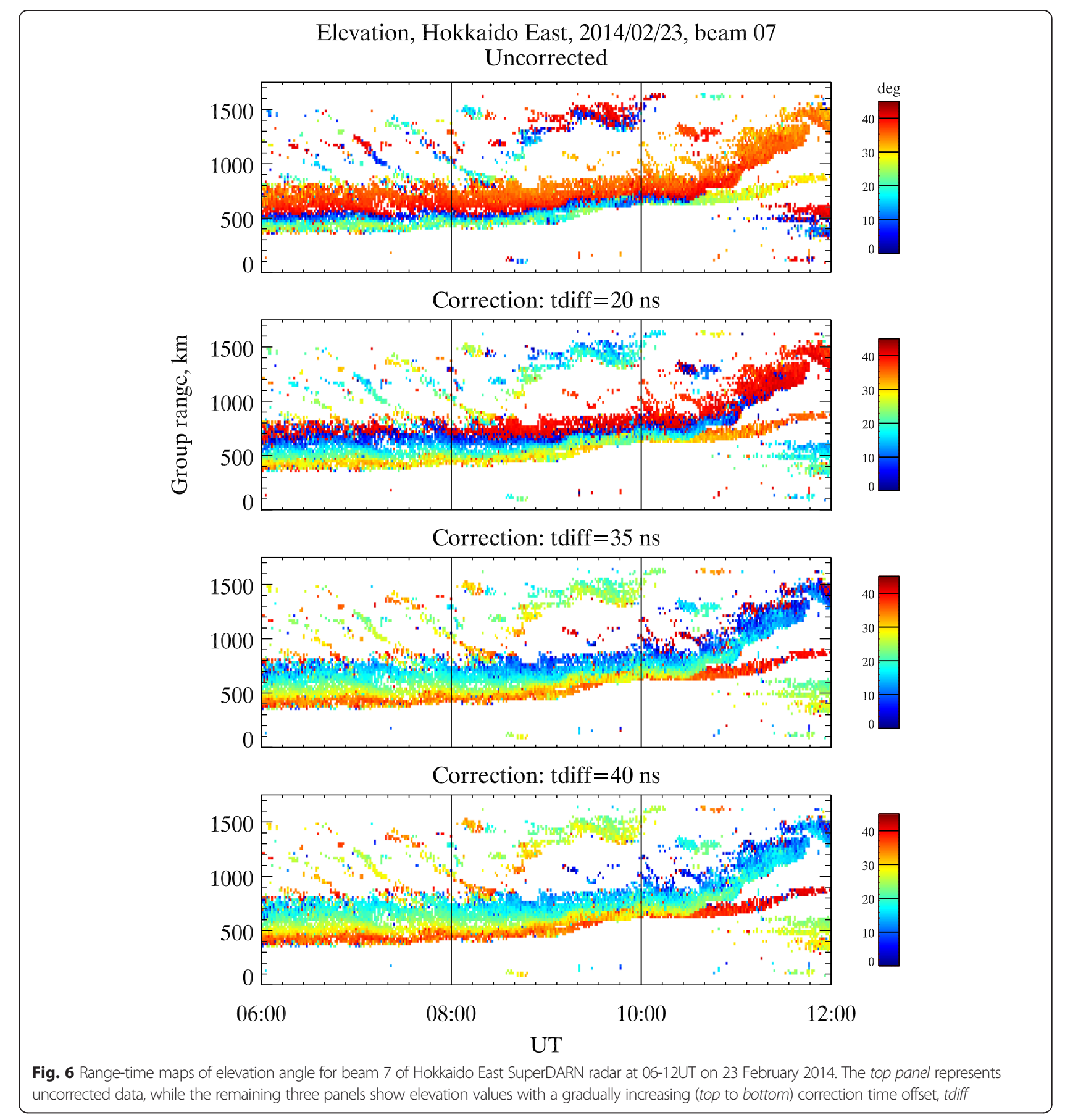

panel shows an uncorrected range-time elevation map for beam 7. In this figure, we used range calculated for GS scatter (defined a half of the group time of flight) so that it roughly corresponds to the ionospheric "reflection" point of the radio wave. Several propagation modes can be identified here: (1) a continuous band of GS covering 400-800 km which starts to move farther away after 9UT; (2) oblique patches of echoes generated by travelling ionospheric disturbances (TIDs) between 800 and $1500 \mathrm{~km}$; (3) a relatively narrow band of ground scatter from the back- lobe clearly seen after 10:30UT between 600 and $900 \mathrm{~km}$. The latter component has been identified based on the criteria for the back-lobe elevation described in Milan et al. (1997). There are also some other patches of echoes which might be related to either ionospheric scatter (11:3012:00UT, $300-600 \mathrm{~km}$ ) or second-hop ground scatter (9:00-10:00UT, 1200-1500 km).

Here, we focus on the main-lobe GS component for which uncorrected elevation exhibits an abrupt change from very low to very high values at $\approx 500 \mathrm{~km}$. This 
change indeed looks unphysical and therefore attributed to the effect of an unaccounted for time/phase delay in the radar's hardware.

In Fig. 7a, we show statistical analysis of the original (uncorrected) data and present two-dimensional range histograms of the phase (top), elevation (middle) and virtual height (bottom). Adequate statistical analysis requires the analysed dataset to be stationary and we therefore limited the analysed interval to 06:00-09:00UT when the propagation conditions remained essentially constant. Furthermore, in order to increase the number of data points, we included in our analysis data from all 16 beams. Phase was automatically confined to the conventional interval corresponding to the elevation values between 0 and $\Delta_{2 \pi}$, as highlighted by red in Fig. 2. While the elevation and virtual height for the uncorrected data show two apparently independent populations, the phase clearly demonstrates a single population "wrapped" around $\Psi_{0}-2 \pi$. By comparing the experimental data with the simulation from Fig. 4, we identified the presence of a phase offset of $\approx 150^{\circ}-160^{\circ}$.

The following plots in Fig. 6 show step-by-step correction by applying a gradually increasing time delay (top to bottom). For $t$ diff $=20 \mathrm{~ns}$, the jump from low to high elevation angles shifts to $\approx 600-650 \mathrm{~km}$. At $t$ diff $=35 \mathrm{~ns}$, the regular discontinuity virtually disappears, but a close look at the farther edge of the ground scatter band during 10:00-12:00UT reveals that multi-point patches of very high elevation are still observed against the low elevation background. By further increasing the time delay to $40 \mathrm{~ns}$ (bottom), the high elevation patches are reduced to isolated pixels, at which stage we assumed that no more correction is needed.

Figure $7 \mathrm{~b}$ shows that once the 40-ns correction has been applied, a single population of echoes is present whose elevation monotonically decreases with range and whose virtual height lies near $280 \mathrm{~km}$. The phase shift required to recover a continuous phase population is close to $160^{\circ}$ which, in terms of time delay, is close to $40 \mathrm{~ns}$ at the Hokkaido East radar operation frequency of $f \approx 11 \mathrm{MHz}$. A distinct but relatively small population in the top right corners of the elevation and phase distributions represents mainly second-hop echoes, while another population at close ranges centred at $\Delta \approx 10^{\circ}$ results from "wrapping" of the returns whose elevation exceeds $\Delta_{2 \pi}$.

While the graphic representation of the elevation-vsrange dependence in Fig. 7 is more convenient for estimating the bulk phase/time offset, its "fine tuning" is more efficiently performed utilising highly contrasting colours for the low and high elevation values in the range-time maps (Fig. 6).

It shall be noticed that our proposed method allows for estimating the phase offset within a $2 \pi$ range so it is generally limited to a single working frequency. As a result, for multi-frequency datasets, the effective time delay (tdiff) has to be determined at each working frequency separately. On the other hand, if for a given radar the phase offset is caused by a frequency-independent time delay, the multifrequency data would allow to evaluate the actual (i.e.

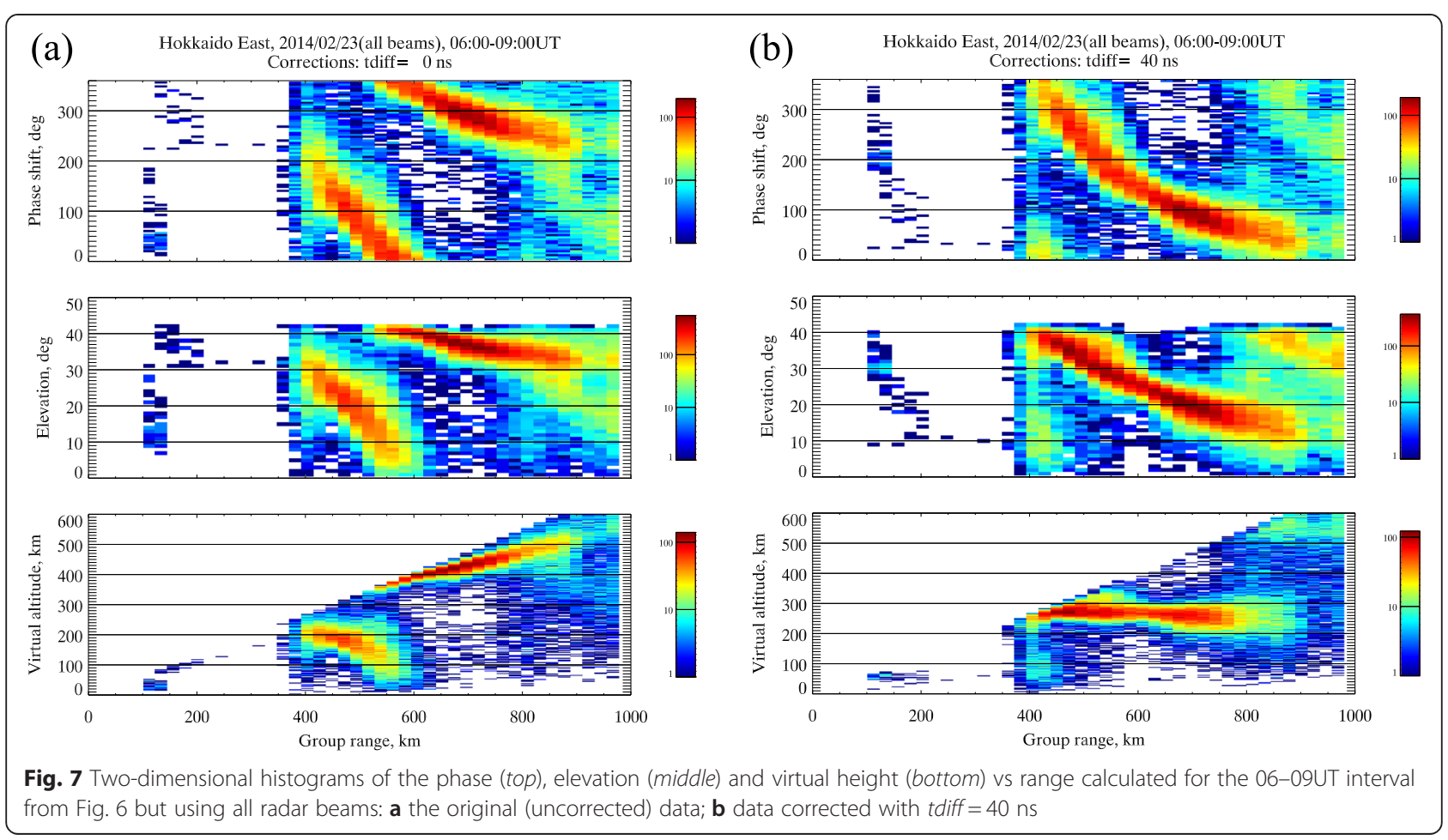


multi-2 $\pi$ ) offset assuming a linear dependence of the phase shift on the radar frequency. For obvious reasons, one has to avoid using data obtained under highly perturbed conditions, and datasets related to different hardware and software updates should be tested separately.

\section{Conclusions}

In this work, we have proposed an empirical method to detect and measure phase offsets in HF interferometry data. The proposed method allows for reliable post-calibration of the historic data, i.e. when the conventional calibration using signal generators is frequently too challenging or nearly impossible, e.g. owing to changes in the hardware. The method is based on analysing the progression of elevation angle with range for ground scatter echoes and uses a gradual adjustment of the phase shift until the observed dependence agrees with that expected from a reflecting ionospheric layer. The theoretical basis for this technique was laid through the simulation of ground scatter propagation characteristics using numerical ray tracing and an analysis of the effects of phase fluctuations on elevation. The method was successfully applied to Hokkaido East SuperDARN radar data producing a reliable estimate of the virtual reflection height. The method also provides the basis to extend diagnostic capabilities of the network to a measure of the background electron density (Ponomarenko et al. 2011b). The use of the proposed technique is not limited to SuperDARN and can be applied to any kind of HF interferometry data.

\section{Abbreviations}

SuperDARN: Super Dual Auroral Radar Network; HF: high-frequency range of electromagnetic emission (3-30 MHz).

\section{Competing interests}

The authors declare that they have no competing interest.

\section{Authors' contributions}

The idea of the phase calibration and its implementation belong to PP. NN and JPSM made substantial contributions to establishing the physical basis for the algorithm and clarifying the details of its implementation. AO and TT performed the bulk of analysis of the Hokkaido East data. All authors read and approved the final manuscript. All authors read and approved the final manuscript.

\section{Acknowledgements}

We would like to thank all the staff who contributed to the HF radar experiment at Hokkaido. This work was supported by Special Funds for Education and Research (Energy Transport Processes in Geospace) of the Ministry of Education, Culture, Sports, Science and Technology of Japan. This work was supported in part by funding from the Government of Canada for a Canada Research Chair (JPSM).

\section{Author details}

'Solar-Terrestrial Environment Laboratory, Nagoya University, Nagoya, Japan. ${ }^{2}$ Institute of Solar-Terrestrial Physics, Siberian Branch of the Russian Academy of Sciences, Irkutsk, Russia. ${ }^{3}$ University of Saskatchewan, Saskatoon, SK, Canada.

Received: 21 March 2015 Accepted: 19 August 2015

Published online: 26 August 2015

\section{References}

André D, Sofko GJ, Baker K, MacDougall J (1998) SuperDARN interferometry: meteor echoes and electron densities from ground scatter. J Geophys Res 103:7003-7015. doi:10.1029/97JA02923.

Blanchard GT, S Sundeen S, Baker KB (2009) Probabilistic identification of highfrequency radar backscatter from the ground and ionosphere based on spectral characteristics. Radio Sci 44:RS5012. doi:10.1029/2009RS004141.

Chisham G, Lester M, Milan SE, Freeman MP, Bristow WA, Grocott A, McWilliams KA, Ruohoniemi JM, Yeoman TK, Dyson PL, Greenwald RA, Kikuchi T, Pinnock M, Rash JPS, Sato N, Sofko GJ, Villain J-P, Walker ADM (2007) A decade of the Super Dual Auroral Radar Network (SuperDARN): scientific achievements, new techniques and future directions. Surv Geophys 28:33-109. doi:10.1007/s10,712-007-9017-8.

Greenwald RA, Baker KB, Dudeney JR, Pinnock M, Jones TB, Thomas EC, Villain J-P, Cerisier J-C, Senior C, Hanuise C, Hunsucker RD, Sofko G, Koehler J, Nielsen E, Pellinen R, Walker ADM, Sato N, Yamagishi H (1995) DARN/SuperDARN: a global view of the dynamics of high-latitude convection. Space Sci Rev 71:761-795

Kelso JM (1964) Radio ray propagation in the ionosphere. McGraw-Hill, New York

Milan SE, Jones TB, Robinson TR, Thomas EC, Yeoman TK (1997) Interferometric evidence for the observation of ground backscatter originating behind the CUTLASS coherent HF radars. Ann Geophys 15:29-39. doi:10.1007/s00585997-0029-y

Ponomarenko PV, St-Maurice J-P, Waters CL, Gillies RG, Koustov AV (2009) Refractive index effects on the scatter volume location and Doppler velocity estimates of ionospheric HF backscatter echoes. Ann Geophys 27:4207-4219. doi:10.5194/angeo-27-4207-2009.

Ponomarenko P, Wiid J, Koustov A, St.-Maurice J-P (2011a) Making sense of SuperDARN elevation: phase offset and variance. In Program and Abstracts for the SuperDARN 2011 Workshop, Thayer School of Engineering, Dartmouth College, Hanover, NH, US, 29 May-03 June 2011

Ponomarenko PV, Koustov AV, St.-Maurice J-P, Wiid J (2011b) Monitoring the F-region peak electron density using HF backscatter interferometry. Geophys Res Lett. doi:10.1029/2011GL049675.

Ruohoniemi JM, Baker KB (1998) Large-scale imaging of high-latitude convection with Super Dual Auroral Radar Network HF radar observations. J Geophys Res 103:20,797-20,811

Virginia Tech SuperDARN group (2015). Range-time plotting on-line tool. http:// vt.superdarn.org/tiki-index.php?page=DaViT+RTP. Accessed on 28 August 2015

\section{Submit your manuscript to a SpringerOpen ${ }^{\odot}$ journal and benefit from:}

- Convenient online submission

Rigorous peer review

- Immediate publication on acceptance

- Open access: articles freely available online

- High visibility within the field

- Retaining the copyright to your article

Submit your next manuscript at $>$ springeropen.com 